\title{
Pulmonary arterial systolic pressure / Acceleration Time of Pulmonary outflow relationship to evaluate the pulmonary ventricle interaction
}

\author{
Walter Serra ${ }^{1}$ and Alfredo Chetta $^{1}$ \\ ${ }^{1}$ University Hospital of Parma
}

May 19, 2020

\begin{abstract}
Background: In pulmonary hypertension $(\mathrm{PH})$, the development of dilatation and the right ventricular failure are signs of accelerated progression of the disease, resulting in an increased risk of cardiac death and right ventricular failure. Even the non-invasive assessment of the systolic blood pressure in the pulmonary artery by echocardiography does not provide a measure of ventricle-pulmonary interaction. Some studies showed that the potential use of echocardiography to evaluate indirectly the pulmonary vascular resistance (PVR) and the acceleration time of pulmonary outflow (ACTPO) could be a good, correlated, indirect measure. Methods: We decided to use a parameter that contained information related to pulmonary artery pressure (sPAP) and pulmonary vascular resistance. We called it PAPs/ACTPO ratio [strength/surface unit]/[time]. We aimed to study this parameter in apparently healthy subjects to code the normal range. From January 2017 to December 2018,60 normal volunteer subjects and 63 patients with systemic scleroderma (Ssc)(60F,3M), 27 with PH and 36 without PH at two-dimensional echocardiographic/Doppler evaluation. Results: In normal subjects, we found a mean sPAP/ACTPO ratio of 0.26 indicative of an optimal pulmonary arterial ventricle coupling and biventricular function. The data derived from the analysis of the patients with Ssc showed that those presenting pre-capillary PH at cardiac catheterization had a sPAP/ACTPO ratio of 0.40 \pm 0.05 . There was a significant correlation between sPAP/ACTPO with Walk Distance (WD) and PVR but not with TAPSE. Interobserver variability was lower than $5 \%$.
\end{abstract}

\section{Key point}

Pulmonary Circulation; Pulmonary Hypertension; Echocardiography; Scleroderma

\section{Introduction}

In pulmonary hypertension $(\mathrm{PH})$, the development of right ventricular (RV) dilation and failure (RVF) are signs of accelerated disease progression that imply an increased risk of cardiac death, $(1,2$,$) . Both \mathrm{PH}$ $(2,3,4)$ and RVF are independently related to clinical worsening, and a cumulative risk prediction exists for RVF and excessive levels of pulmonary arterial systolic pressure (PAPs).

Although the non-invasive estimation of pulmonary artery systolic pressure (PAPs) by Doppler echocardiography is well established $(5,6,13)$, it does not measure the right ventricular-arterial coupling (RV- $A C$ ) and the pulmonary vascular resistance (PVR). Some studies showed the potential use of echocardiography to assess the PVR $(12,13)$. The Acceleration Time of Pulmonary Outflow (ACTPO) should be an indirect measure of this parameter (14). Assessment of RV dimensions, geometry, and function is affected by the complex chamber shape, requiring assumptions through noninvasive techniques, however there is agreement in considering echocardiography as the most immediate and simple approach to obtain reliable markers of RV systolic function in cardiopulmonary disorders. Among the echo-derived variables used for RV systolic 
function assessment, the tricuspid annular plane systolic excursion (TAPSE) is easily obtainable, reproducible, and highly predictive of poor outcome in $\mathrm{PH}$ patients $(6,10,11$,). A combined assessment would provide a more physiology-based information (16). Accordingly, we hypothesized that the relationship between ACTPO (time) and sPAP (developed pressure) could be used and proposed as an in vivo expression of the right ventricular coupling and pulmonary pressure-time relationship, and that a combination of these variables in a ratio might better disclose prognosis compared with either single variable.

\section{Methods}

\section{Patients.}

From January 2017 to December 2018, 123 consecutive patients, 60 volunteer normal (N) and 63 with systemic scleroderma $(\mathrm{Ssc}),(60 \mathrm{~F}, 3 \mathrm{M})$, were screened for study enrolment at the time of referral for a clinically indicated hemodynamic and functional assessment. Patients underwent a two-dimensional echocardiographic/Doppler evaluation. The inclusion criteria of scleroderma patients were: history, signs, symptoms, and treatment in accordance with American College of Rheumatology (ACR)/European League Against Rheumatism (EULAR current guidelines(17). Subjects with significant valvular heart disease (moderate to severe stenosis/regurgitation), hypertrophic or infiltrative cardiomyopathy, pericardial diseases, unstable ischemic heart disease (acute coronary syndrome or recent myocardial infarction $<60$ days before selection) were excluded. Other exclusion criteria were: inability to perform $6 \mathrm{MWT}$ and/or poor acoustic window. Enrolled patients were monitored in this prospective, observational study. Imaging was performed using a Philips IE33 and a 5.2-MHz transducer (Philips Medical Systems, Andover, MA). A two-dimensional Doppler examination was performed through a protocol using views specifically designed to optimize the RV imaging (18). To obtain the TAPSE, the apical four-chamber view was used, and an M-mode cursor was placed through the lateral tricuspid annulus in real time. The TAPSE was measured as the peak excursion of the tricuspid annulus (millimeters) from the end of diastole to end systole. The ACTPO pulmonary artery flow was measured by PW-Doppler through the pulmonary valve jet from the short axis view, as the interval (msc) between the onset of ejection and the peak flow velocity Fig. 1 to five beats.

RV systolic pressure was determined from the tricuspid regurgitation (TR) jet velocity using the simplified Bernoulli equation, and combining this value with an estimate of the right atrial pressure by the diameter and collapsibility of the inferior vena cava, that was added to the calculated gradient to yield sPAP. Because subjects had no significant RV outflow tract or pulmonic valve obstruction, RV systolic pressure was considered equal to PAPs. All measurements were performed by two senior researchers who read the recordings in a blinded manner and without knowledge of the clinical diagnosis.

Fig 2 shows the values of sPAP/time vs. time. In both cases it is noted that at very low times, the power required at the heart is very high. Moreover, the curves have an articulated trend that has a maximum corresponding to a time of high resistance: the lower curve refers to the normal case because of the lower value ( $\mathrm{sPAP} /$ time is 0.19 ), that reaches the maximum for $\mathrm{t} \sim 68 \mathrm{~ms}$.

\section{Right heart catheterization}

The patients were submitted to femoral right heart catheterization using a $7 \mathrm{~F}$ catheter thermodilution to evaluate the systolic, mean and capillary pulmonary pressure and calculate the trans-pulmonary gradient $(\mathrm{TPG}=\mathrm{PAPm}-\mathrm{PCWPm}), \mathrm{PVR}($ dynes $\mathrm{Sm}-5=\mathrm{TPG} / \mathrm{CO} \times 80)$ and cardiac output $(\mathrm{CO}) . \mathrm{PH}$ was defined as a mean PAP $(\mathrm{PAPm})>25 \mathrm{~mm} / \mathrm{Hg}$ and a Pulmonary Capillary Wedge Pressure $(\mathrm{PCWP})<15 \mathrm{~mm} / \mathrm{Hg}$ (1), although new limits have been proposed ad a new haemodynamic definitions and updated clinical classification of Pulmonary Hypertension have emerged from the $6^{\text {th }}$ World Symposium of PH (19).

Event monitoring and endpoints. Patients were monitored for worsening dyspnea and for cardiac mortality via hospital and outpatient medical chart review.

\section{Statistical analysis}

The primary objective of the study was to assess the range of SPAP/ACTPO ratio in healthy subjects and 
in patients with scleroderma and PH. The second objective was to correlate the sPAP/ACTPO ratio and the hemodynamic parameters (sPAP, PVR) at the right heart catheterization. Data are expressed as mean values $\pm \mathrm{SD}$ unless otherwise stated. Pearson's correlation coefficient and Bland-Altman analysis were used to compare sPAP, ACTPO, sPAP/ACTPO ratio and TAPSE with PAPs, PVR and NYHA class. Stepwise forward multiple regression analysis allowed the weighting of the independent effects of the potential determinants on an independent variable. The null hypothesis was rejected when $\mathrm{p}$ was $>0.05$. The sPAP/ACTPO ratio was measured by two sonographers blinded to the clinical data in order to assess the interobserver variability.

\section{Ethics statement}

This study was conducted in accordance with Good Clinical Practice and the current version of the revised Declaration of Helsinki. Written informed consent was obtained from each patient prior to enrollment.

\section{Results}

Baseline demographic, clinical, and haemodynamic characteristics of patients with scleroderma and normal arterial pressure are summarized in Table $\mathbf{1}$, while baseline demographic, clinical, and haemodynamic characteristics of patients with scleroderma and $\mathrm{PH}$ are summarized in

\section{Table 2.}

Age is not significantly different between the two groups (t-student).PAP/ACTPO is weakly related to age, both in the healthy $(\mathrm{r}=0,42, \mathrm{p}=0,014)$ and in the scleroderma patients $(\mathrm{r}=0,41, \mathrm{p}=0,079=>)$. Interobserver variability was 5\%. 36 patients showed no $\mathrm{PH}$ and 27 with $\mathrm{PH}$ associated with Ssc, at a two-Dimensional echocardiographic/Doppler evaluation. sPAP/ACTPO ratio showed high correlations with both the terms used for its calculation: $\mathrm{sPAP}(\mathrm{R}=0,88, \mathrm{P} 0,001)$ and ACTPO $(\mathrm{R}=-0,90, \mathrm{P} 0,001)$.

In normal subjects the mean sPAP/ACTPO ratio was $0.19 \pm 0,063$, indicative of an optimal afterload, good pulmonary arterial ventricle coupling, where pulmonary pressure, biventricular function and NYHA class were normal. We then evaluated this parameter in patients with PH associated with Ssc.; the data derived from the 20 Ssc patients with pre-capillary PH to cardiac catheterization had a sPAP/ACTPO ratio of 0.40 \pm 0.05 Fig. 3 . There was a significant correlation between sPAP/ACTPO and Walk Distance (WD) and PVR, but not with TAPSE, perhaps because sPAP/ACTPO has a greater correlation with afterload, thus its ratio might be a better approach to afterload in itself, cardiac output and arterial ventricle coupling. Fig $4 \mathrm{~A}, 4 \mathrm{~B}$

\section{Discussion}

Right ventricular arterial coupling (RV-AC) implies efficiency of energy transfer from the ventricle to the arterial load and has arisen as a novel parameter to assess the adequate matching between contractility and afterload. It is calculated as the ventricular elastance (Ees) over arterial elastance (Ea)ratio (Ees/Ea). The complicated invasive assessment (it is obtained from volume-pressure curves) has lead to factible and practical non invasive approaches with echocardiography and cardiac magnetic resonance. The most robust RV-AC echo parameter was proposed by Guazzi et al (20), and combined in a ratio TAPSE/sPAP (numerator presenting RV performance and a denominator an afterload parameter). A good invasive correlation was shown with the gold standard for RV-AC (ventricular elastance / arterial elastance). Moreover, clinical and prognostic information has been provided in the last 5 years with publications in different pulmonary hypertension populations. A recent study of the different echo parameters that could provide a non invasive approach of RV-AC in SSc was published(21). The sPAP/ACTPO ratio represents the power required for the heart to push the blood into the pulmonary artery (cardiac power). In fact, dimensionally PAPs $=$ Energy/Volume and power = Energy/Time: through the Bernoulli theorem the pressure is linked to the kinetic energy, to the density of the blood, i.e. to the kinetic energy per unit of volume. It could be argued that the Bernoulli theorem is an approximation too strong to describe a viscous fluid like the blood. In this case, however, the effect of the resistance due to the viscosity is already taken into account by inserting the ACTPO variable. Good conditions are those of low resistance, corresponding to a long acceleration time. In this case, 
the heart provides low energy to the blood and therefore it works less. Doppler echocardiography derived ACTPO assesses the blood flow velocity characteristics in the RV outflow tract in response to changes in RV mechanical performance and pulmonary vascular load. Recent works demonstrated that ACTPO provides a reliable estimate of invasive vascular resistance $(22,23,24)$. The relationship of sPAP/ACTPO with invasive measures of pulmonary hemodynamic was not explored in this cohort and because PH can exert a significant load to the right ventricle, a non-invasive index to characterize ventricular-vascular coupling that shows all aspects of RV afterward will be useful for risk stratification and long-term monitoring. From the values we obtained we observed, for each value considered, an increase in SPAP/ACTPO following a decrease in ACTPO that could be due to a reduction in cardiac output and to an increase in pulmonary vascular resistance. In our study, there was no correlation between sPAP/ACTPO and TAPSE ; however, there was a significant correlation with PVR and the WD, perhaps because this parameter is more related to the afterload, cardiac output and arterial ventricle coupling. Ventricular work decreases as the ratio decreases in normal subjects by $0.19 \pm 0.063$. In subjects with $\mathrm{PH}$, the high ratio tending to 1 highlights the great work that the right ventricle must perform against high pulmonary vascular resistance.

Both, sPAP and ACTPO are also indirect afterload measures, so its ratio could be a better approach to both afterload and RV-AC performance per se.

This result leads to consider the possibility of measuring the efficiency of the right heart power before and after a pharmacological or surgical intervention, such as pulmonary thromboendarterectomy. It is well established that there is a poor RV adaptation to overload in Ssc $(25,26)$, which is also linked to a complex pathophysiology that could be identified by the sPAP/ACTPO ratio.

\section{Limitations}

Our study was single-center and relatively small. Moreover, echocardiographic examinations and RHC were not done simultaneously, possibly affecting the results. We found SPAP/ACTPO values that correlated with pre capillary forms of pulmonary hypertension, but these should be better evaluated in studies that use cardiac catheterism in all patients. Finally, the determination of sPAP/ACTPO was slightly limited by the availability of pulmonary Doppler signals.

\section{Conclusions}

The sPAP/ACTPO ratio is a new parameter that may be indicative of pulmonary arterial ventricle coupling, pulmonary vascular afterload and interaction, both in normal subjects and in patients with or without $\mathrm{PH}$ with Ssc.

Key points : - Although non-invasive assessment of pulmonary artery systolic pressure (PAPs) by Doppler echocardiography is well established, this is not a measure of ventricle-lung interaction, right ventricle-arterial coupling (RV-ac) and pulmonary vascular resistance (PVR). Some studies (Abbas, Serra) have shown the potential use of echocardiography to assess PVR $(12,13)$ and the acceleration time of pulmonary outflow $(\mathrm{ACTPO})$, should be an indirect measure of this parameter. $\mathrm{PVR}=\mathrm{CO} /$ deltaP.

To get a measure of the ventricle-pulmonary interaction, we used a parameter that contained information related to pulmonary pressure, therefore given by a ratio of a force per unit area and a parameter that was an indicator of pulmonary vascular resistance.

- The study provides three messages : First, the primary goal was to simply measure the parameter in healthy subjects and this was done.

- We tested the parameter in patients with scleroderma without and with pulmonary hypertension, to give normality limits that were well coded.

- In subjects with $\mathrm{PH}$, the high ratio tending to 1 , highlights the great work that the right ventricle must perform against high lung resistance. This result leads to consider the possibility of measuring the efficiency of the right heart power before and after a pharmacological or surgical intervention. 
Abbreviations : PH: pulmonary hypertension; BMI: body mass index, defined as weight in kilograms divided by the square of height in meters; CAD: coronary artery disease; CI: confidence interval; DPG: diastolic pulmonary gradient;

LV: left ventricle;

mPAP: mean pulmonary artery pressure; sPAP: systolic pulmonary artery pressure; PCWP: pulmonary capillary wedge pressure; PVR: pulmonary vascular resistance;

RA: right atrium;

RV: right ventricle;

RV FAC: right ventricular fractional area change; TAPSE: tricuspid annular plane systolic excursion;

TPG: transpulmonary gradient;

TR: tricuspid regurgitation;

ACTPO: acceleration time of pulmonary outflow;

SSc: systemic sclerosis;

RHC: right heart catheterization;

WD: Walk Distance

\section{REFERENCES}

1. Nazzareno Galie ${ }^{*}$,Marc Humbert*, Jean-Luc Vachiery, Simon Gibbs , Irene Lang, Adam Torbicki, Gerald Simonneau et al ... The ESC/ERS Guidelines for the diagnosis and treatment of pulmonary hypertension. European Heart Journal (2016)37, 67-119

2. Kawut SM, Taichman DB, Archer-Chicko CL, Palevsky HI, Kimmel SE. Hemodynamics and survival in patients with pulmonary arterial hypertension related to systemic sclerosis. Chest 2003;123:344-350.

3. Mathai SC, Sibley CT, Forfia PR, Mudd JO, Fisher MR, Lechtzin N, Boyce D, et al. Tricuspid annular plane systolic excursion is a robust outcome measure in scleroderma Associated pulmonary arterial hypertension. J Rheumatol 2011;38(11):2410-2418.

4. Campo A, Mathai SC, Le Pavec J, Zaiman AL, Hummers LK, Boyce D, Housten T, et al. Hemodynamic predictors of survival in scleroderma-related pulmonary arterial hypertension. Am J Respir Crit Care Med 2010;182:252-260.

5. Tedford RJ, Mudd JO, Girgis RE, Mathai SC, Zaiman AL, Housten-Harris T, Boyce D, et al. Right ventricular dysfunction in systemic sclerosis-associated pulmonary arterial hypertension. Circ Heart Fail 2013;6:953-963.

6. Badesch DB, Champion HC, Sanchez MA, Hoeper MM, Loyd JE, Manes A, McGoon M, et al. Diagnosis and assessment of pulmonary arterial hypertension. J Am Coll Cardiol 2009;54:S55-S66.

7. McLaughlin VV, Archer SL, Badesch DB, Barst RJ, Farber HW, Lindner JR, Mathier MA, et al. ACCF/AHA 2009 expert consensus document on pulmonary hypertension: a report of the American College of Cardiology foundation task force on expert consensus documents and the American Heart Association developed in collaboration with the American College of Chest Physicians; American Thoracic Society, Inc.; and the Pulmonary Hypertension Association. J Am Coll Cardiol 2009;53:1573-1619.

8. Mukerjee D, St George D, Coleiro B, Knight C, Denton CP, Davar J, Black CM, Coghlan JG. Prevalence and outcome in systemic sclerosis associated pulmonary arterial hypertension: application of a registry approach. Ann Rheum Dis 2003;62:1088-1093. 
9. Hachulla E, Gressin V, Guillevin L, Carpentier P, Diot E, Sibilia J, Kahan A, et al. Early detection of pulmonary arterial hypertension in systemic sclerosis: a French nationwide pulmonary prospective multicenter study. Arthritis Rheum 2005;52:3792-3800.

10. Steen VD, Medsger TA. Changes in causes of death in systemic sclerosis, 1972-2002. Ann Rheum Dis 2007;66: 940-944.

11. Franey L, Abbas A Marwick T, Vlahos A, Serra W et al. Assessment of markedly pulmonary vascular resistance by echocardiography. ACC March 2013 ISSN: 0735-1097.

12. Abbas AE, Franey LM, Marwick T, Maeder MT, Kaye DM, Vlahos AP, Serra W, Al-Azizi K, Schiller NB, Lester SJ. Noninvasive assessment of pulmonary vascular resistance by Doppler echocardiography. J Am Soc Echocardiogr. 2013;26(10):1170-1177.

13. Serra W, Chetta A, Santilli D, Mozzani F, Dall'Aglio PP, Olivieri D, Cattabiani MA, Ardissino D, Gherli T. Echocardiography may help detect pulmonary vasculopathy in the early stages of pulmonary artery hypertension associated with systemic sclerosis. Cardiovasc Ultrasound. 2010; 8:25.

14. Levy PT, Patel MD, Groh G, Choudhry S, Murphy J, Holland MR, et al. Pulmonary artery acceleration time provides a reliable estimate of invasive pulmonary hemodynamics in children. J Am Soc Echocardiog 2016;29: 1056-65.

15. Wang Y-C, Huang CH, Tu Y-K. Pulmonary hypertension and pulmonary artery acceleration time: a systematic review and meta-analysis. J Am Soc Echocardiogr 2018;31:201-10. 10.

16. Koestenberger M, Grangl G, Avian A, Gamillscheg A, Grillitsch M, Cvirn G, et al. Normal reference values and $\mathrm{Z}$ scores of the pulmonary artery acceleration time in children and its importance for the assessment of pulmonary hypertension. Circ Cardiovasc Imaging 2017;10: e005336.

17. Van den Hoogen F, Khanna D, Fransen J, Johnson SR, Baron M, Tyndall A. 2013 classification criteria for systemic sclerosis: an American college of rheumatology/European league against rheumatism collaborative initiative. Ann Rheum Dis . 2013 Nov. 72(11):1747-55.

18. Patrizio Lancellotti, Christophe Tribouilloy, Andreas Hagendorff, Bogdan A. Popescu, Thor Edvardsen, Luc A. Pierard, Luigi Badano, Jose L. Zamorano, Recommendations for the echocardiographic assessment of native valvular regurgitation: an executive summary from the European Association of Cardiovascular Imaging. European Heart Journal - Cardiovascular Imaging, Volume 14, Issue 7, July 2013, Pages 611-644,

19. Simonneau $\mathrm{G}^{1,2}$, Montani $\mathrm{D}^{1,2}$, Celermajer $\mathrm{DS}^{3}$, Denton $\mathrm{CP}^{4}$, Gatzoulis $\mathrm{MA}^{5}$, Krowka $\mathrm{M}^{6}$, Williams $\mathrm{PG}^{7}$, Souza $\mathrm{R}^{8}$. Haemodynamic definitions and updated clinical classification of pulmonary hypertension. Eur Respir J. 2019 Jan 24;53(1). pii: 1801913. doi: 10.1183/13993003.01913-2018. Print 2019 Jan.

20. Guazzi M, Bandera F, Pelissero G, Castelvecchio S, Menicanti L, Ghio S, et al. Tricuspid annular plane systolic excursion and pulmonary arterial systolic pressure relationship in heart failure: an index of right ventricular contractile function and prognosis. Am J Physiol Heart Circ Physiol 2013;305: H1373-81.

21. Sarah French, Myriam Amsallem, Nadia Ouazani, Shufeng Li, Kristina Kudelko,Roham T. Zamanian, Francois Hadda, and Lorinda Chung, Non-invasive right ventricular load adaptability indices inpatients with scleroderma-associated pulmonary arterial hypertension. Pulmonary Circulation 2018; 8(3) 1-11].

22. Koestenberger M, Grangl G, Avian A, Gamillscheg A, Grillitsch M, Cvirn G, et al. Normal reference values and $\mathrm{Z}$ scores of the pulmonary artery acceleration time in children and its importance for the assessment of pulmonary hypertension. Circ Cardiovasc Imaging 2017;10: 05336.

23. Vonk-Noordegraaf A, Westerhof BE, Westerhof N. The relationship between the right ventricle and its load in pulmonary hypertension. J Am Coll Cardiol 2017;69:236-43.16.

24. Milnor WR. Arterial impedance as ventricular afterload. Circ Res 1975;36: 565-70. 
25. Gargani L, Voilliot D, D'Alto M, Agoston G, Moreo A, Serra W, Pieri F, Mori F, Wierzbowska-Drabik K, Matucci-Cerinic M, Moggi-Pignone A. Pulmonary Circulation on the Crossroads Between the Left and Right Heart in Systemic Sclerosis: A Clinical Challenge for Cardiologists and Rheumatologists. Heart Fail Clin 2018;14(3):271-281.

26. Christian Nagel MD , Alberto M. Marra MD, Nicola Benjamin MSc, Norbert Blank MD, Antonio Cittadini MD, PhD , Gerry Coghlan MD And Ekkehard Grünig MD . Reduced Right Ventricular Output Reserve in Patients With Systemic Sclerosis and Mildly Elevated Pulmonary Artery Pressure. Arthritis and Reumatology 2019 doi: 10.1002/art.40814

\section{Figure legends}

\section{Figure 1}

TR was evaluated in the apical four-chamber view, the parasternal short-axis view at the level of the aortic valve, and the RV inflow view. Its severity, using the jet area/right atrial area method, was graded as follows: mild, if the regurgitant jet area/right atrial area was $<19 \%$; moderate, if $20-40 \%$; severe, if [?]41\%. The ACTPO Pulmonary artery flow was measured by PW-Doppler through the pulmonary valve jet from the short axis view, as the interval between the onset of ejection and the peak flow velocity. The data was corrected for heart rate $(\mathrm{ACTPO} / \mathrm{HRx} 100)$. The equation (TRV/TVI $\left.\mathrm{RVOT}^{*} 10\right)+0.16$ has been shown to provide a non-invasive correlate of PVR.

\section{Figure 2}

Figure 2 shows the values of PAPS/time vs. time. In both cases it is noted that at very low times, the power required at the heart is very high. Moreover, the curves have an articulated trend that has a maximum corresponding to a time of high resistance: the lower curve refers to the normal case because of the lower value (PAPS/time is 0.19 ), that reaches the maximum for $\mathrm{t} \sim 68 \mathrm{~ms}$.

\section{Figure 3}

The first data derived from the analysis of the 20 Ssc patients show that those presenting pre-capillary $\mathrm{PH}$ at cardiac catheterization have a PAPs/ACTPO ratio $>$ of $0.40+-0.05$. There was a significant correlation between PAPs/ACTPO and WD, but not with PVR, perhaps because this parameter has a greater correlation with cardiac output and arterial ventricle coupling.

\section{Figure 4}

A Correlation between PAPs/ACTPO and PVR; BCorrelation between PAPs/ACTPO and WD.

\section{Tables}

Table 1. Baseline demographic, clinical, and haemodynamic characteristics of patients with Ssc and normal arterial pressure.

Table 2. Baseline demographic, clinical, and haemodynamic characteristics of patients with Ssc and PH.

\section{Hosted file}

PAPSACTPO ratio Figure ECHO.docx available at https://authorea.com/users/323827/articles/ 452306-pulmonary-arterial-systolic-pressure-acceleration-time-of-pulmonary-outflowrelationship-to-evaluate-the-pulmonary-ventricle-interaction

\section{Hosted file}

PAPSACTPO TAB.docx available at https://authorea.com/users/323827/articles/452306-pulmonaryarterial-systolic-pressure-acceleration-time-of-pulmonary-outflow-relationship-toevaluate-the-pulmonary-ventricle-interaction 